East African Medical Journal Vol. 78 No. 11 November 2001

LOWER GENITAL TRACT INFECTIONS AMONG PREGNANT WOMEN: A REVIEW

W. Marai, MD, Associate Professor, Department of Obstetrics and Gynaecology, Jimma University, Jimma, P.O. Box 13253, Addis Ababa, Ethiopia.

\title{
LOWER GENITAL TRACT INFECTIONS AMONG PREGNANT WOMEN: A REVIEW
}

\author{
W. MARAI
}

\section{ABSTRACT}

Objectives: To determine the prevalence of lower genital tract infections, discuss briefly common maternal foetal complications associated with them and assess the usefulness of diagnostic algorithms in their management among pregnant women in the developing countries.

Data source: Articles published in English language since 1987 were looked through MEDLINE and OVID using key words supplemented by manual search in libraries except when full text of a subject was accessible via internet.

Study selection: Original and review articles addressing genital tract infections, associated complications and diagnostic evaluation in pregnant women were included. Emphasis was given to articles reported from developing countries.

Data extraction: A total of thirty five articles were retrieved and reviewed for information on the performance of diagnostic algorithms, prevalence rates and adverse maternal-foetal effects of lower genital tract infections in pregnancy.

Data synthesis: Lower genital tract infections are very common among apparently healthy looking pregnant women with an overall prevalence of 40-54\%. Specific pathogens that were isolated from the vagina and/or cervix of asymptomatic pregnant women include: $C$. albicans (14-42\%), T. vaginalis $(11-20 \%)$, C. trachomatis $(7-31 \%)$, . gonorrhoea $(0.5-14 \%)$ and group $B$ streptococcus (4-25\%). Untreated, genital tract infections in pregnant women may result in: foetal loss, preterm labour, preterm birth, premature rupture of the membranes, low birthweight, eye and lung damage in the newborn. Although the feasibility is good, the performance of clinical algorithms in the evaluation and management of lower genital tract infections is worse in pregnant women and better results are achieved for vaginal infections than cervical infections.

Conclusion: Routine screening for clinically important pathogens should be considered during antenatal service. There is a need to develop simple, cheap and reliable laboratory tests and better clinical algorithms for the diagnosis of reproductive tract infections among pregnant women.

\section{INTRODUCTION}

Lower genital tract infections (LGTIs) are serious risk factors for significant maternal and neonatal morbidity and mortality (1-3). They may be associated with premature rupture of the membranes (PROM), preterm labour (PTL), preterm birth, low birthweight (LBW), various types of foetal, neonatal infections and puerperal sepsis $(3,4)$. In certain areas of the world, cervico-vaginal infection with sexually transmitted organisms is as high as $50 \%$ among pregnant women attending the public sector antenatal service(5). It is now well documented that the acquisition of human immunodefficiency virus (HIV) is facilitated by sexually transmitted infections (STIs). There is evidence that effective treatment of STIs reduces the incidence of HIV(6). For all these reasons, the management of STIs in developing countries is a priority as part of reproductive health programmes and in the campaign against the expansion of HIV. Pregnancy and antenatal visits constitute an opportunity for STIs management in women. This may help to reduce infectivity, maternal foetal complications and give good opportunity to teach and reduce risk taking behaviour for STIs.

There are different useful laboratory methods to detect genital tract infections in women. However, they are expensive and not widely available in the developing countries. To overcome this, the WHO proposed a diagnostic algorithm based on clinical signs, symptoms and risk assessment to be used at the primary health care level. The usefulness of these methods is currently under investigation in different countries. This review has been conducted to determine the prevalence of LGTIs among pregnant women, to asses the usefulness of diagnostic algorithms in the management of LGTIs among pregnant women in the developing countries and discuss some maternal-foetal complications associated with LGTIs.

\section{MATERIALS AND METHODS}

Literature was searched using MEDLINE and OVID with key words: Pregnancy, prevalence, infections, preterm labour, cervix and vagina. Manual search was done in libraries when the full text of a subject in a journal was not accessible via the 
internet. Standard obstetric and gynaecologic books were consulted when necessary. Review was limited to pathogens for which data are accumulating from the developing world. Therefore, this review does not include discussions on human papilloma viruses, herpes simplex virus and genital ulcers.

\section{RESULTS}

Prevalence of LGTIs: A wealth of data has started to accumulate on the prevalence and pattern of reproductive tract infections in the developing countries. In general the prevalence of LGTIs among pregnant women in this countries is high. Among none symptomatic 646 women in Liberville, Gabon, the prevalence rate was eleven per cent for cervical infections and $40 \%$ for vaginal infections(7). In Burkina Faso, West Africa, among 645 antenatal clinic attendees, the prevalence rate for any reproductive tract infection was found to be $43 \%$, and among these women $32 \%$ presented at least one STIs(8). Studies done in Kenya on apparently "low risk " pregnant women following antenatal care revealed a prevalence rate of $54 \%$ for any vaginal infection and $11 \%$ for any cervical infection(9).

Tabie 1

Prevalence of LGTIs among asymptomatic pregnant women

\begin{tabular}{|c|c|c|c|c|c|c|}
\hline \multirow[b]{2}{*}{ Author } & \multicolumn{4}{|c|}{ Organisms isolated (\%) } & \multirow[b]{2}{*}{$\mathrm{CA}$} & \multirow[b]{2}{*}{ GBS } \\
\hline & NG & $\mathrm{CT}$ & TV & BV & & \\
\hline Meda et al $(8)$ & 1.3 & 3.1 & 14 & 13 & 14 & NA \\
\hline Thomas et al $(9)$ & 2.4 & 8.8 & 20 & 21 & 26 & NA \\
\hline Bourgeois et al(7) & 1.9 & 10 & 11 & 23 & 31 & NA \\
\hline McGregor et al(17) & 0.5 & 7 & - & 19 & 42 & 3.5 \\
\hline Cotch et al (28) & NA & NA & 19 & $\mathbf{N A}$ & NA & NA \\
\hline Garland et al(27) & $\mathrm{NA}$ & NA & NA & NA & NA & 13 \\
\hline Nash et al(1) & 14 & A & 15 & NA & NA & NA \\
\hline McGregor et al(32) & 0.6 & 8.5 & NA & 31 & 21 & 5.8 \\
\hline Duncan et all(1) & 10 & 31 & 20 & NA & NA & NA \\
\hline
\end{tabular}

$\mathrm{NA}=$ Not available

GBS $=$ Group B streptococcus

Mayaud et al(10) reported a rate of $68 \%$ for any cervical or vaginal infections among 660 women reporting for routine antenatal care in urban setting in Mwanza, Tanzania. The rate of any cervical infection was $7.4 \%$ which was not different from other studies mentioned above. A study done by the same author on symptomatic (vaginal discharge) pregnant women in the same region demonstrated the prevalence of cervical infection to be eight per cent which is also close to the figures for asymptomatic women in the above series. A study of 342 routine antenatal clinic attendees by Duncan et al(11) in Addis Ababa, Ethiopia showed $72 \%$ of them had serological evidence of two or more STIs.

Diagnostic evaluation of LGTIs: Lower genital tract infections can be diagnosed with considerable ease using different ancillary or definitive diagnostic aids. A wet mount of vaginal secretions detects $65-70 \%$ of culture proven trichomoniasis and 70-80\% of gram stain confirmed BV $(1,12)$. Among symptomatic women about $85 \%$ of candidiasis is demonstrated by culture. Gram stain from endo-cervical specimen detects approximately $60 \%$ of gonorrhoea diagnosed by culture. Leucocyte detection using leucocyte esterase test (LET) on cervical swab or clean caught urine might be of help in the evaluation of LGTIs(13). More advanced tests such as polymerase chain reaction tests and enzyme linked immuno-assays are also available to diagnose CT, mycoplasmas and viral infections. Chlamydia trachomatis and N. gonorrhoea can be diagnosed using cell culture and Thyer Martin agar media respectively. Papanicolaou smear may be useful in the detection of TV similar to that of wet mount but not reliable or generally not available in many developing countries(1). Various clinical or bedside aids have been suggested to effectively recognise and treat LGTIs in areas where accurate but expensive tests are not available $(13,14)$. Taking the issue of cost and feasibility into consideration, in 1991, the WHO introduced a syndromic approach based on the complaints of the people who suffer from genital symptoms for STIs management. The flow chart for vaginal discharge syndrome is shown in Figure 1. The algorithm has been evaluated in various modifications with different bed side tests such as LET

Figure 1

WHO flow chart for vaginal discharge syndrome with risk assessment

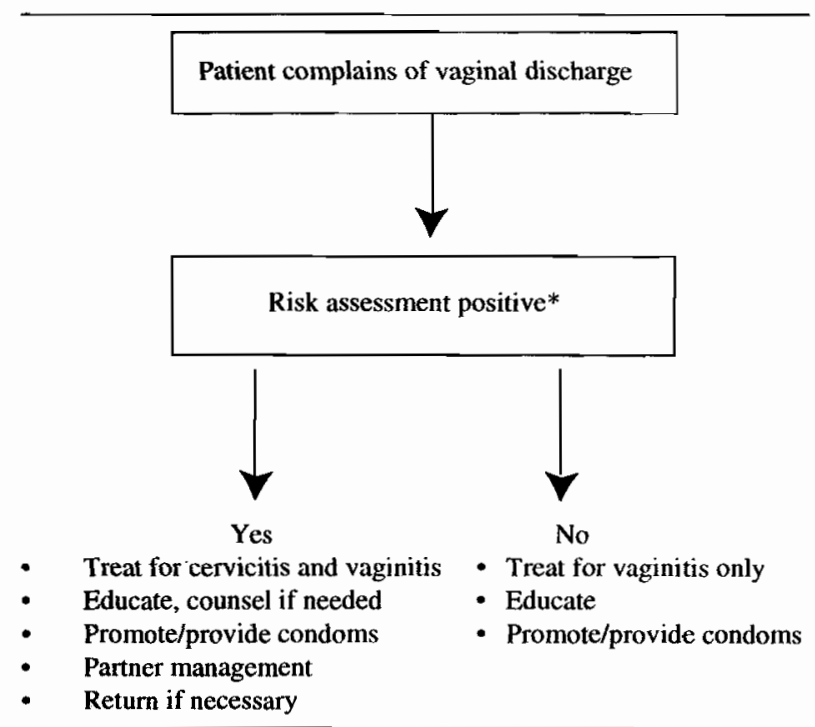

*Partner symptomatic or any two of: age $<21$ years, single, more than one partner or new partner in the past three months

and cervical swab test (muco-pus on white cotton). Although the feasibility is good, it is said to be $70 \%$ sensitive and $70 \%$ specific at best(15). Reports indicate that the performance is worse in pregnant women and better results are achieved for vaginal infections than cervical infections $(8,9)$. The specificity, sensitivity and positive predictive values from different studies on pregnant women following antenatal care are given in Table 2. 
Table 2

Sensitivity, specificity and positive predictive values of WHO and various local clinical algorithms used in the evaluation of vaginal discharge in pregnant women

\begin{tabular}{|c|c|c|c|}
\hline & Sensitivity $(\%)$ & Specificity (\%) & $\begin{array}{c}\text { Positive predictive } \\
\text { value }(\%)\end{array}$ \\
\hline Meda et al $(8)$ & 80 & 50 & 7 \\
\hline Thomas et al(9) & 19 & 75 & 10 \\
\hline Bourgeois et al(7) & 74 & 50 & 10 \\
\hline Vuylsteke et al(29) & 72 & 74 & 16 \\
\hline Mayaud et al(10) & 10 & 92 & 10 \\
\hline
\end{tabular}

Table 3

Summary of selected adverse pregnancy outcome measures associated with LGTIs

\begin{tabular}{|c|c|c|c|c|c|c|c|}
\hline Pathogen & Abortion & SB & PTL & PROM & LBW & $\begin{array}{l}\text { Endomyometritis } \\
\text { chorio amnionitis }\end{array}$ & $\begin{array}{l}\text { Neonatal } \\
\text { infection }\end{array}$ \\
\hline $\operatorname{Ng}(1)$ & + & - & + & + & + & + & + \\
\hline $\mathrm{Ct}(1,7)$ & - & + & + & + & + & + & + \\
\hline Mycoplasmas (22) & + & + & + & - & -- & + & + \\
\hline \multicolumn{8}{|l|}{ Group B } \\
\hline Streptoccocus (27) & - & - & + & + & - & + & + \\
\hline $\mathrm{BV}(1,5)$ & - & - & + & + & - & + & - \\
\hline TV (18) & - & - & + & + & - & - & - \\
\hline
\end{tabular}

$S B=$ still birth

\section{IMPLICATION OF LGTIS FOR PREGNANT WOMEN AND THEIR OFFSPRINGS}

Pregnancy complications. Lower genital tract infections are found to be associated with different pregnancy complications. Bacterial vaginosis and NG are implicated in spontaneous abortion, preterm labour, preterm birth and premature rupture of the membranes(1,16-18). Chlamydia trachomatis, group B streptococci, and TV appear to be associated with premature rupture of the membranes and preterm labour(18,19). In the light of this evidence and the fact that $C$. trachomatis and $N$. gonorrhoea are STIs with public health relevance, the Center for Disease Control recommended screening pregnant women for these pathogens(20). Bacterial vaginosis is the most prevalent cause of vaginal discharge in the developed countries and also common among pregnant women in the developing countries. It may cause amniotic fluid infections and postpartum sepsis( 16). Lower genital tract infection with candida species has not been found to be linked to adverse pregnancy outcome(21).

Adverse foetal and neonatal outcomes: Lower genital tract infections of sexually transmitted nature carry a risk of vertical transmission resulting in neonatal morbidity. Neisseria gonorrhoea and C. trachomatis are associated with ophthalmia neonatorum, corneal ulceration and blindness if not treated or prevented. Chlamydia trachomatis and Ureaplasma urealyticum are incriminated in pneumonia and possibly chronic lung disease in the latter case(22,23). Bacterial vaginosis, C. trachomatis and $N$. gonorrhoea may cause LBW(1,19,24). Studies from the developed countries show colonisation of the female genital tract with group B streptococcus associated with early onset neonatal sepsis(21).

Maternal complications: Puerperal infections are important causes of postpartum morbidity and mortality. Studies have indicated that the rate of post caesarean wound infections and postpartum endometritis increase because of infection with BV $(3,16)$. Chlamydia trachomatis is implicated as a cause of late onset, indolent endometritis in a third of women who had antepartum chlamydial cervicitis(25). Colonisation of the lower genital tract with group B streptococcus and Mycoplasma homnis has been reported to be linked with increased risk of postpartum infections( 3,22$)$. Untreated gonococcal cervicitiscan progress to chorio-amnionitis and postpartum endo-myometritis(1).

\section{DISCUSSION}

It has been suggested that due to biomedical, behavioural and societal factors, reproductive tract infections are widespread in the third world. Some of the factors influencing female reproductive tract infections may include: urbanisation with loosening traditional restraints on sexual activity, low level of education and female financial self-insufficiency making them dependant on males, promoting prostitution to generate income. In all of the African studies, the prevalence both of vaginal and cervical infection (N. gonorrhoea and/or $C$. trachomatis) are high(7-10). Meda et al(8) from Burkina Faso and Thomas et al(9) from Kenya found over $85 \%$ of their study subjects were married; however, the prevalence rate in the groups were the same(7-9). In earlier studies 
from Kenya it was suspected that females in stable relationships are probably infected from their male sex partners(26). This may be the explanation for the above finding that stable married women had a rate of infection nearly similar to women who were not in stable relationship. The prevalence of colonisation of female genital tract with group B streptococcus was reported to be as high as $25 \%$ in some series from the developed world(27). Prenatal colonisation is a risk factor for early onset neonatal sepsis. Such infections are usually due to gram negative enterococci (coliforms) in developing countries like Ethiopia(personal observation). Research done previously linked this organism with: premature rupture of the membranes and PTL $(17,18)$. But there is recent evidence that there may not be such an association(27). There is lack of information on the subject from less developed part of the world. Trichomonas vaginalis and BV are frequently recovered from the lower genital tracts of pregnant women as shown by different studies $(7,8,28)$ which calls for early recognition and appropriate management.

The diagnosis and management of LGTIs is a challenge for both planners and health service providers in certain areas of the world. These is because there are no simple, cheap, reliable diagnostic methods to handle these disorders. Relatively simple, feasible flow charts have been proposed for use in resource poor areas $(14,29)$. But their sensitivity and specificity perform inconsistently across groups of patients studied(8-10,30). They actually do worse in pregnant women than in non-pregnant women and better for vaginal discharge than cervical infections. Therefore more research should be done to develop cost effective, reliable diagnostic tools and screening clinical algorithms that can work for different risk groups. Most of the evidence of the association between LGTIs and adverse pregnancy outcome comes from the developed world. While LGTIs in developing world are still important, their contribution to pregnancy outcome in those settings must be studied separately. Maternal genital tract colonisation with specific organisms has inconsistently been associated with PTL, preterm birth PROM, and LBW $(17,18)$. A varying number of patients with unexplained $P$ TL have positive aminiotic fluid culture $(0-30 \%)(31)$. It was indicated that up to $80 \%$ of early preterm births are associated with intra-amnoiotic infections. There are many trials of antibiotic therapy to prevent or delay preterm delivery, but they allow no definite conclusion as to the efficacy due to variation in time of initiation of therapy, use of different antibiotics or other unrecognised factors $(18,32)$. Bacterial vaginosis is said to be common in preterm delivery independent of other risk factors(24). In randomised double blind study on pregnant women at risk of preterm delivery with $\mathrm{BV}$, administration of metronidazole and erythromycin at a mean gestational age of 29.9 weeks was shown to significantly reduce the rate of preterm delivery(33). A consensus seems to emerge that high-risk women need to be screened and treated for the disorder early in pregnancy $(16,20)$. Further studies are needed on the applicability of this evidence to low risk women.
An impressive patho-physiologic mechanism involving cytokines has been forwarded to explain how sub-clinical or histologic chorioamnionitis might initiate PTL. In this model bacterial products (lipoplysacharides, peptidoglycans) activate macrophages of the decidua and polymorphonuclear leucocytes are recruited. This leads to a rise in IL-1B, arachidonic acid and prostaglandins-E2. $\mathrm{IL}-1 \beta$ stimulates production of TNF $\alpha$, IL-6, and IL-8 which are elevated during term and preterm labour. But there is a debate whether these events precede or follow labour such that exposed membranes and decidua during cervical dilatation might lead to similar biochemical changes(18,34,35). Recognition and treatment of LGTIs, particularly the sexually transmitted ones is very important. Because, untreated, they might predispose to puerperal infections which contribute to the disproportionate maternal death toll in the poor countries. Besides, they also contribute to the high rate of foetal loss and neonatal morbidity and mortality.

In conclusion, the prevalence of LGTIs among pregnant women in the developing world is high and calls for commitment to routine evaluation and appropriate intervention in antenatal clinics. There is lack of simple, cheap and reliable diagnostic tools for the evaluation and management of LGTIs. Available clinical approaches have low sensitivity, specificity and do not appear to serve in all settings. Lower genital tract infections affect pregnancy outcome adversely. The effective control of LGTIs that are STIs has both individual and public health relevance. A research is needed to develop cheaper, simple, reliable diagnostic tools and clinical algorithms for use in different settings in resource poor areas. The magnitude and nature of maternal, foetal and neonatal complication of LGTIs in the developing countries need to be addressed well.

\section{ACKNOWLEDGEMENTS}

To Dr Kevin O'Reilly for reviewing the manuscript and International Association for Maternal and Neonatal Health (IAMANEH) for financial assistance.

\section{REFERENCES}

1. Wasserheit J.N. The significance and scope of reproductive tract infections among women. Int. J. Gynecol. Obstet. 1998; (suppl 3): 74:145-167.

2. Berman S.M., Harrison H.R. and Boyce W.T., et al. Low birth weight, prematurity, and postpartum endometritis in adolescents. J. Amer. Med. Ass. 1987; 257:1189.

3. Bernson, Hammiss H.A. and Martens M.G. et al. Bacteriological findings of post-caesarean endometritis in adolescents. Obstet. Gynec. 1990; 75: 627.

4. Fliegner J.R. and Garland S.M. Perinatal mortality in Victoria Australia: Role of group B steptococcus. Amer. J. Obstet. Gynec. 1990; 163:1609-1611.

5. Wilkinson D. Sexually transmitted infections (editorial) Genitourinary Med. 1997; 73:427-428.

6. Grosskurth H., Mosha F. and Todd, et al. Impact of improved treatment of sexually transmitted diseases on HIV infection in rural Tanzania: Randomized controlled trial. Lancet. 1995; 346:530536 